\title{
Separation and screening of short-chain chlorinated paraffins in environmental samples using comprehensive two-dimensional gas chromatography with micro electron capture detection
}

\author{
Dan Xia $\cdot$ Lirong Gao $\cdot$ Shuai Zhu $\cdot$ Minghui Zheng
}

Received: 11 July 2014 / Revised: 14 September 2014 / Accepted: 19 September 2014 /Published online: 11 October 2014

(C) Springer-Verlag Berlin Heidelberg 2014

\begin{abstract}
Short-chain chlorinated paraffins (SCCPs) are highly complex technical mixtures with thousands of isomers and numerous homologs. They are classified as priority candidate persistent organic pollutants under the Stockholm Convention for their persistence, bioaccumulation, and toxicity. Analyzing SCCPs is challenging because of the complexity of the mixtures. Chromatograms of SCCPs acquired using onedimensional (1D) gas chromatography (GC) contain a large characteristic "peak" with a broad and unresolved profile. Comprehensive two-dimensional $\mathrm{GC}(\mathrm{GC} \times \mathrm{GC})$ shows excellent potential for separating complex mixtures. In this study, $\mathrm{GC} \times \mathrm{GC}$ coupled with micro electron capture detection $(\mu \mathrm{ECD})$ was used to separate and screen SCCPs. The chromatographic parameters, including the GC column types, oven temperature program, and modulation period, were systematically optimized. The SCCP congeners were separated into groups using a DM-1 column connected to a BPX-50 column. The SCCP congeners in technical mixtures were separated according to the number of chlorine substituents for a given carbon chain length and according to the number of carbon atoms plus chlorine atoms for different carbon chain lengths. A fish tissue sample was analyzed to illustrate the feasibility of the $\mathrm{GC} \times \mathrm{GC}-\mu \mathrm{ECD}$ method in analyzing biological samples. Over 1,500 compounds were identified in the fish extract, significantly more than were identified using $1 \mathrm{D}$ GC. The detection limits for five selected SCCP congeners were between 1 and $5 \mathrm{pg} / \mathrm{L}$ using the $\mathrm{GC} \times \mathrm{GC}$ method, and these were significantly lower than those achieved using $1 \mathrm{D}$ GC. This method is a good choice for analysis of SCCPs in
\end{abstract}

D. Xia $\cdot$ L. Gao $(\triangle) \cdot$ S. Zhu $\cdot$ M. Zheng

State Key Laboratory of Environmental Chemistry and

Ecotoxicology, Research Center for Eco-Environmental Sciences,

Chinese Academy of Sciences, No. 18, Shuangqing Road, Haidian

District, Beijing 100085, China

e-mail: gaolr@rcees.ac.cn environmental samples, exhibiting good separation and good sensitivity.

Keywords Comprehensive two-dimensional gas chromatography · Micro electron capture detection · Short chain chlorinated paraffins $\cdot$ Environmental samples

\section{Introduction}

Chlorinated paraffins (CPs) are highly complex technical mixtures with a chlorination degree between 30 and $70 \%$ and carbon chain lengths of $\mathrm{C}_{10}-\mathrm{C}_{13}$ (short-chain CPs, SCCPs), $\mathrm{C}_{14}-\mathrm{C}_{17}$ (medium-chain CPs), or $\mathrm{C}_{18}-\mathrm{C}_{30}$ (longchain CPs) [1, 2]. Technical formulations of CPs have been used widely for many years, as additives in lubricants and cutting fluids and as flame retardants in plastics and sealants [3]. SCCPs have received considerable attention from environmental scientists worldwide in recent years because of their ability to undergo long-range transport, their persistence in the environment, their bioaccumulation in food webs, and their toxicity to aquatic organisms [4]. SCCPs have been classed as priority hazardous substances in the European Water Framework Directive, and are also under review by the Stockholm Convention as a potential "new" category of priority candidate persistent organic pollutants [5]. SCCPs have been found at high concentrations in a variety of environmental matrices, such as marine sediment $(0.21-1.17 \mu \mathrm{g} /$ g) [6] and biota $(480-3,300 \mu \mathrm{g} / \mathrm{g})$ [7]. CPs have been produced in the European Union and the USA, Canada, China, and other countries since the 1930s, and the amount of CPs produced in China (where they remain in use) has continued to increase. Attention should, therefore, be given to the concentrations of SCCPs in environmental matrices so that the risks they pose to humans through exposure to them can be assessed. However, data on the concentrations of SCCPs in 
the environment are very limited, largely because of the complexity involved in analyzing them quantitatively.

The analysis of SCCPs in environmental samples is far from being well established [8]. The quantification of SCCPs is an extremely challenging task because SCCP mixtures contain thousands of different individual isomers, homologs, diastereomers, and enantiomers, and the different congeners have similar chromatographic retention characteristics. Additionally, interferences caused by the constituents of the real environmental matrices make the accurate determination of SCCPs difficult. SCCPs have been determined in environmental samples using a number of types of instrumental methods, including gas chromatography (GC) with an electron capture detector (ECD) and GC coupled with low- or high-resolution mass spectrometry [9-11]. However, singlecolumn GC methods cannot separate the individual SCCP congeners and isomers, and the chromatograms produced contain a characteristic broad feature containing a large number of peaks corresponding to coeluted compounds. The accurate quantification of SCCPs has still not been achieved, and better analytical methods are urgently needed.

Comprehensive two-dimensional (2D) $\mathrm{GC}(\mathrm{GC} \times \mathrm{GC})$ is a separation technique that uses two chromatographic columns with different polarities. The columns are connected in series through an interface called the modulator that allows an entire sample to be separated in two dimensions $[12,13]$. GC $\times \mathrm{GC}$ gives better overall resolution, total peak capacity, and selectivity than does GC, and it has been indisputably shown in recent years that $\mathrm{GC} \times \mathrm{GC}$ is a very powerful technique for analyzing a variety of highly complex mixtures. The ECD is a classic GC detector that is very sensitive to polyhalogenated compounds. ECDs have been widely used in $\mathrm{GC} \times \mathrm{GC}$ systems because ECDs offer a high scanning rate (more than $20 \mathrm{~Hz}$ ) and the ability to accurately characterize the narrow peaks produced by a $\mathrm{GC} \times \mathrm{GC}$ system [14]. A micro-ECD $(\mu \mathrm{ECD})$ has been developed that has a much smaller inner volume (only $150 \mu \mathrm{L}$ ) than an ECD, meaning that it can avoid the band broadening problems that occur when using a conventional $\mathrm{ECD}$ in a $\mathrm{GC} \times \mathrm{GC}$ system. A combination of $\mathrm{GC} \times \mathrm{GC}$ with a $\mu \mathrm{ECD}$ has recently given very good results, and it has been successfully used to analyze polychlorinated biphenyls, polychlorinated dibenzo- $p$-dioxins and dibenzofurans, and toxaphene in complex environmental matrices [15-19]. Korytár et al. [20] studied the analysis of CPs by $\mathrm{GC} \times \mathrm{GC}$ coupled with time-of-flight mass spectrometry. It provides some information in terms of ordered structures, i.e., group and subgroup separation. The information on $\mathrm{GC} \times \mathrm{GC}$ analysis of SCCP mixtures as presented in this article is still limited and resolution is not complete. And to the best of our knowledge, no more research has been performed on the separation of SCCPs using $\mathrm{GC} \times \mathrm{GC}$.

In this work, we analyzed SCCPs in technical mixtures and biological samples using comprehensive $\mathrm{GC} \times \mathrm{GC}$ with a $\mu \mathrm{ECD}$. Different column sets, GC oven temperature programs, modulation periods, carrier gas flow rates, and other instrumental parameters were tested and optimized. The method that was developed was used to analyze the five common technical SCCP mixtures. The SCCP congeners in technical mixtures were separated according to the number of chlorine substituents for a given carbon chain length and according to the number of carbon atoms plus chlorine atoms for different carbon chain lengths. Also, the method's applicability to biological samples was demonstrated by analyzing a fish sample. The limits of detection (LODs) for the selected SCCP congeners analyzed by $\mathrm{GC}-\mathrm{ECD}$ and $\mathrm{GC} \times \mathrm{GC}-\mu \mathrm{ECD}$ methods were compared.

\section{Experimental}

Standard materials and reagents

Pesticide analysis grade solvents were purchased from J.T.Baker (Phillipsburg, NJ, USA). Three SCCP mixtures $\left(\mathrm{C}_{10}-\mathrm{C}_{13}\right.$ containing $51 \%, 55.5 \%$, and $63 \%$ chlorine; $100 \mathrm{ng} / \mu \mathrm{L}$ solutions in cyclohexane; $100 \%$ pure; Dr. Ehrenstorfer, Augsburg, Germany) were used to determine the optimum $\mathrm{GC} \times \mathrm{GC}-\mu \mathrm{ECD}$ conditions. Technical standard mixtures (10 $\mathrm{ng} / \mathrm{mL}$ in cyclohexane) of SCCPs with different carbon chain lengths and chlorine contents $\left(\mathrm{C}_{10} 44.82 \%\right.$, $50.18 \%, 55.00 \%, 60.09 \%$, and $65.02 \%$ chlorine; $\mathrm{C}_{11}$ $45.50 \%, 50.21 \%, 55.20 \%$, and $60.53 \%$ chlorine; $\mathrm{C}_{12}$ $45.32 \%, 50.18 \%, 55 \%, 65.08 \%$, and $69.98 \%$ chlorine; and $\mathrm{C}_{13} 44.9 \%, 50.23 \%, 55.03 \%, 59.98 \%$, and $65.18 \%$ chlorine) were purchased from Dr. Ehrenstorfer. A CP " $\mathrm{C}_{10}$ mix 1" standard solution (containing 1,2,5,6,9,10hexachlorodecane, 1,2,5,6,9,10-hexachlorodecane, 1,2,5,6,9pentachlorodecane, 2,5,6,9-tetrachlorodecane, and 1,2,9,10tetrachlorodecane) and a $\mathrm{CP}$ " $\mathrm{C}_{10}$ mix 2" standard solution (containing 1,2,5,6,9-pentachlorodecane, 1,2,4,5,9,10hexachlorodecane, 1,2,4,5,6,9,10-heptachlorodecane, 2,3,4,5,6,7,8,9-octachlorodecane, and 1,2,3,4,5,6,7,8,9nonachlorodecane) were obtained from Dr. Ehrenstorfer.

\section{Sample preparation}

A fish (Triplophysa siluroides) sample collected from a river in China was used to validate the method that was developed. The extraction and cleanup method described by Gao et al. [21] was used to prepare the sample. The dry fish tissue sample $(2.0 \mathrm{~g})$ was homogenized with anhydrous $\mathrm{Na}_{2} \mathrm{SO}_{4}$ $(15.0 \mathrm{~g})$, spiked with $10 \mathrm{ng}$ of ${ }^{13} \mathrm{C}_{10}$-trans-chlordane, then extracted with a mixture of dichloromethane and hexane (1:1 $\mathrm{v}: \mathrm{v})$ in an accelerated solvent extraction apparatus (ASE350; Dionex, Sunnyvale, CA, USA). The extraction was done in three 5 -min cycles at $100^{\circ} \mathrm{C}$ and $1.03 \times 10^{4} \mathrm{kPa}$, followed by a 
10 -min static extraction, then a flush volume of $60 \%$ and $\mathrm{a}_{2}$ purge time of $60 \mathrm{~s}$ were applied. Acidified silica gel $(37.5 \%$ by weight sulfuric acid) was added to the extract to remove lipids and other potentially interfering compounds. The sample was then concentrated and further cleaned using a chromatographic column (10-mm inner diameter) containing (from bottom to top) $5.0 \mathrm{~g}$ of activated alumina, $2.0 \mathrm{~g}$ of basic silica gel, $2.0 \mathrm{~g}$ of activated silica gel, $5.0 \mathrm{~g}$ of acidified silica gel (30\% by weight sulfuric acid), and $4.0 \mathrm{~g}$ of anhydrous $\mathrm{Na}_{2} \mathrm{SO}_{4}$. The extract was eluted from the column with $40 \mathrm{~mL}$ of hexane and $100 \mathrm{~mL}$ of a 1:1 (v:v) mixture of hexane and dichloromethane. The extract collected was concentrated to about $50 \mu \mathrm{L}$ under a gentle $\mathrm{N}_{2}$ stream. An injection internal standard (10 ng of $\varepsilon$-hexachlorocyclohexane) was added and mixed completely with the extract before $\mathrm{GC} \times \mathrm{GC}$ analysis was performed.

\section{$\mathrm{GC} \times \mathrm{GC}$ instrument}

The first GC column was a $30 \mathrm{~m}$ long, $0.25 \mathrm{~mm}$ inner diameter, and $0.25 \mu \mathrm{m}$ film thickness DM-1 column (Dikma, Lake Forest, CA, USA). We determined the optimum column set for the analysis by testing three possible second GC columns: BPX50 (50\% phenyl polysilphenylene-siloxane phase; $1 \mathrm{~m}$ long, 0.10-mm inner diameter, $0.10-\mu \mathrm{m}$ film thickness; SGE, Austin, TX, USA), this combination of GC columns later being called DM-1×BPX50; BPX70 (70\% cyanopropyl polysilphenylene-siloxane phase; $1 \mathrm{~m}$ long, 0.10-mm inner diameter, 0.10- $\mu \mathrm{m}$ film thickness; SGE), which is later called DM- $\times$ BPX70; and DB-WAX [poly(ethylene glycol) phase, $1 \mathrm{~m}$ long, 0.10-mm inner diameter, $0.10-\mu \mathrm{m}$ film thickness; Agilent Technologies, Santa Clara, CA, USA], which is later called DM- $1 \times$ DB-WAX.

The GC $\times$ GC system consisted of an 7890 GC system (Agilent Technologies, Santa Clara, CA, USA) equipped with a dual-jet two-stage loop, a ZX2 thermal modulator (Zoex, Houston, TX, USA), and a $\mu$ ECD. The principles and characteristics of the modulator are described elsewhere [22]. The GC oven temperature was initiated at $100{ }^{\circ} \mathrm{C}$, which was held for $2 \mathrm{~min}$, then increased at $10^{\circ} \mathrm{C} / \mathrm{min}$ to $180{ }^{\circ} \mathrm{C}$, which was held for $2 \mathrm{~min}$, and then increased at $1.5^{\circ} \mathrm{C} / \mathrm{min}$ to $260{ }^{\circ} \mathrm{C}$, which was held for $10 \mathrm{~min}$. A mini press-fit connector (Zoex) was used to connect the first and second GC columns. Liquid nitrogen was used to cool the nitrogen gas that was used to provide the cold pulses. Helium gas (99.999\% pure) was used as the carrier gas at an inlet pressure of $276 \mathrm{kPa}$ and a constant flow rate of $1.0 \mathrm{~mL} / \mathrm{min}$. Injections $(1 \mu \mathrm{L})$ were performed in splitless mode using an Agilent 7683 autosampler, and the injector temperature was $260{ }^{\circ} \mathrm{C}$. The hot air temperature in the modulator was $280{ }^{\circ} \mathrm{C}$, and a 300-ms hot air pulse was used to desorb the trapped material from the modulator. The modulation period was $6 \mathrm{~s}$. A high detector temperature and the highest possible makeup gas flow rate $(150 \mathrm{~mL} / \mathrm{min})$ were used. GC Image version 2.1 (Zoex) was used to analyze the contour plots of the $\mathrm{GC} \times \mathrm{GC}$ chromatograms.

\section{Results and discussion}

Selection of the column set

Because comprehensive $\mathrm{GC} \times \mathrm{GC}$ uses two $\mathrm{GC}$ columns, selecting a suitable combination of columns is essential for successfully separating the target analytes [22, 23]. Three $\mathrm{GC} \times \mathrm{GC}$ column combinations were tested by analyzing SCCP standard mixtures. The combinations used each consisted of a nonpolar column followed by a polar column because this combination provided a broad scope for separating the analytes. Relative to a reversed column set (i.e., the nonpolar column followed by the polar column), orthogonal separation can be obtained in a nonpolar× polar column set. A narrow bore capillary column with a nonpolar $100 \%$ dimethylpolysiloxane (DM-1) phase was selected as the first GC column, to separate the SCCPs according to their boiling points. A moderately polar column (BPX-50; $50 \%$ phenyl polysilphenylene-siloxane phase), a highly polar column (BPX-70; $70 \%$ cyanopropyl polysilphenylene-siloxane phase), and a strongly polar column [DB-WAX; poly(ethylene glycol) phase] were tested as potential second columns. Figure 1 shows the 2D chromatograms obtained for the SCCPs using each of the three column sets. Overall, the separation and resolution of the SCCPs were better when using the $\mathrm{GC} \times \mathrm{GC}$ system with all three column combinations than when using a one-dimensional (1D) GC system even though the different column combinations gave different elution patterns and separations between particular congeners. As can be seen from chromatogram A in Fig. 1, the DM-1 $\times$ BPX70 combination gave better separation than was achieved using 1D GC, but neighboring groups of SCCPs were not fully separated, and no effective separation was achieved overall. The maximum temperature for the BPX-70 column was $250{ }^{\circ} \mathrm{C}$, so more time than we allowed may have been required to separate the constituents effectively using this column at the relatively low temperatures that were possible. Some of the analytes may not even have been eluted from the column. Furthermore, the high polarity of the BPX-70 column meant that the SCCP congeners were strongly retained, which could cause broadening of the chromatographic peaks and poorer sensitivity. The $2 \mathrm{D}$ chromatogram obtained using the DM- $1 \times$ BPX-50 column combination contained clearly ordered SCCP peaks, and good orthogonal separation of SCCP groups was achieved. This was a distinct improvement over the separation achieved using 1D GC, which gave a characteristic unresolved and broad feature. The separation achieved during the analytical run using the DM-1 $\times$ BPX-50 column 
combination was found to be sufficient for separating the SCCPs even in real sample extracts. An orthogonal ordered structure can clearly be seen in chromatogram B in Fig. 1. The SCCP congeners were eluted regularly in the $2 \mathrm{D}$ chromatogram, with no wraparound in the second-dimension separation, and group type separation was successfully achieved. However, the DM- $1 \times$ DB-WAX column combination retained the SCCPs too strongly for an acceptable degree of separation to be achieved, because the DB-WAX column had a very polar stationary phase, and wraparound was found to have occurred. The expected separation power was not achieved with the DM- $1 \times$ DB-WAX column set.

The DM- $1 \times$ BPX-50 column set was therefore chosen for subsequent experiments because it offered the group type separation of the SCCP congeners in technical mixtures in a 2D chromatogram. This column combination can be recommended for the analysis of SCCPs in other studies.

\section{Optimization of the GC oven temperature program}

Optimizing the GC oven temperature program is very important because of the influence it has on the $\mathrm{GC} \times \mathrm{GC}$ separation that will be achieved [24]. The first- and second-dimension GC separations were conducted in the same oven in our $\mathrm{GC} \times \mathrm{GC}$ system, so the first-dimension separation was performed at a linearly increasing temperature and the seconddimension separation (which was achieved in a matter of seconds) was effectively almost isothermal. In general, the oven temperature in a $\mathrm{GC} \times \mathrm{GC}$ system should increase rather slowly, at between 0.5 and $5{ }^{\circ} \mathrm{C} / \mathrm{min}$, to give relatively broad peaks in the first dimension, which allows between 2.5 and four modulations (the required range for satisfactory interpretation to be possible) during each first-dimension peak [25]. The separation achieved using the selected DM- $1 \times$ BPX-50 column combination was evaluated using three oven temperature programs, each with a different heating rate $(X=1,1.5$, and $\left.5{ }^{\circ} \mathrm{C} / \mathrm{min}\right)$ in the main separation phase. The temperature program was as follows: $100^{\circ} \mathrm{C}$, held for $2 \mathrm{~min}$, increased at $10^{\circ} \mathrm{C} / \mathrm{min}$ to $180^{\circ} \mathrm{C}$, then increased at $X^{\circ} \mathrm{C} / \min$ to $260{ }^{\circ} \mathrm{C}$, which was held for $10 \mathrm{~min}$. A heating rate of $1{ }^{\circ} \mathrm{C} / \mathrm{min}$ gave the best separation of the SCCP congeners, as can be seen in the chromatogram of a technical SCCP mixture shown in Fig. 2a, but this rate, of course, gave the longest analysis time. In addition, many peaks suffered wraparound and occurred with peaks in a different modulation cycle when the heating rate was $1{ }^{\circ} \mathrm{C} / \mathrm{min}$ (e.g., compounds eluted at around 5-6 s in the second dimension would be coeluted with those eluted at $1-2 \mathrm{~s}$ in the next modulation cycle). This suggests that the modulation period used when the heating rate was $1{ }^{\circ} \mathrm{C} / \mathrm{min}$ was insufficient for the compounds entering the second column to be eluted within that modulation cycle, causing them to be eluted in the next modulation cycle. A heating rate of $1.5^{\circ} \mathrm{C} / \mathrm{min}$ gave good chromatographic separation of the SCCPs and an acceptable analysis time, as can be seen in Fig. 2b. The relatively short run time not only gave a higher laboratory output than could be achieved using a heating rate of $1{ }^{\circ} \mathrm{C} / \mathrm{min}$, but less liquid nitrogen was consumed in the modulation process. A heating rate of $5{ }^{\circ} \mathrm{C} / \mathrm{min}$ gave significantly poorer separation of the SCCP congeners than was achieved at a heating rate of $1.5{ }^{\circ} \mathrm{C} / \mathrm{min}$, and the ability of the $\mathrm{GC} \times \mathrm{GC}$ system to separate the SCCP congeners was actually almost lost when using such a high heating rate. Therefore, the optimum heating rate was determined to be $1.5^{\circ} \mathrm{C} / \mathrm{min}$, and the oven temperature program was as follows: $100{ }^{\circ} \mathrm{C}$, held for $2 \mathrm{~min}$, increased at $10^{\circ} \mathrm{C} / \mathrm{min}$ to $180{ }^{\circ} \mathrm{C}$, which was held for $2 \mathrm{~min}$, then increased at $1.5^{\circ} \mathrm{C} / \mathrm{min}$ to $260^{\circ} \mathrm{C}$, which was held for $10 \mathrm{~min}$.

\section{Optimization of the modulation period}

The modulation period has a profound influence on the separation achieved when using a $\mathrm{GC} \times \mathrm{GC}$ system. It is widely recognized that wraparound can be a problem when analyzing matrix-rich environmental samples because peaks of coeluted
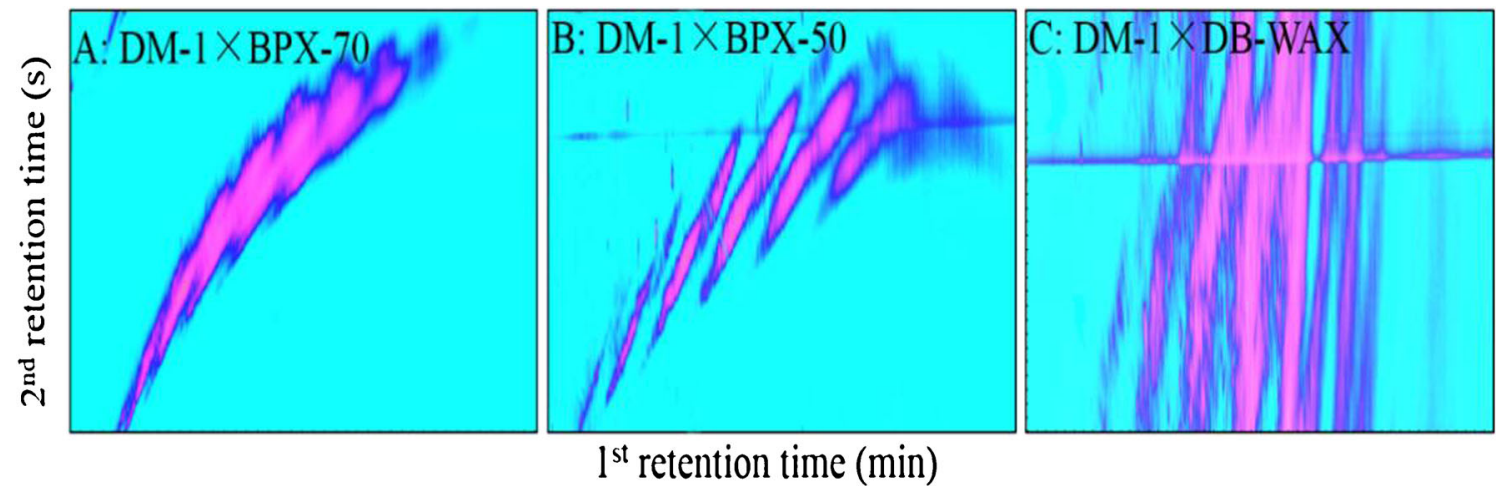

Fig. 1 Two-dimension gas chromatography $(\mathrm{GC} \times \mathrm{GC})$-micro electron capture detection $(\mu \mathrm{ECD})$ chromatograms of a technical $\mathrm{C}_{10}-\mathrm{C}_{13}$ shortchain chlorinated paraffin (SCCP) mixture with a $55 \%(\mathrm{w} / \mathrm{w})$ chlorine content using three different column combinations: a DM-1 column

followed by a BPX-70 column (a), a DM-1 column followed by a BPX-50 column (b), and a DM-1 column followed by a DB-WAX column (c) 

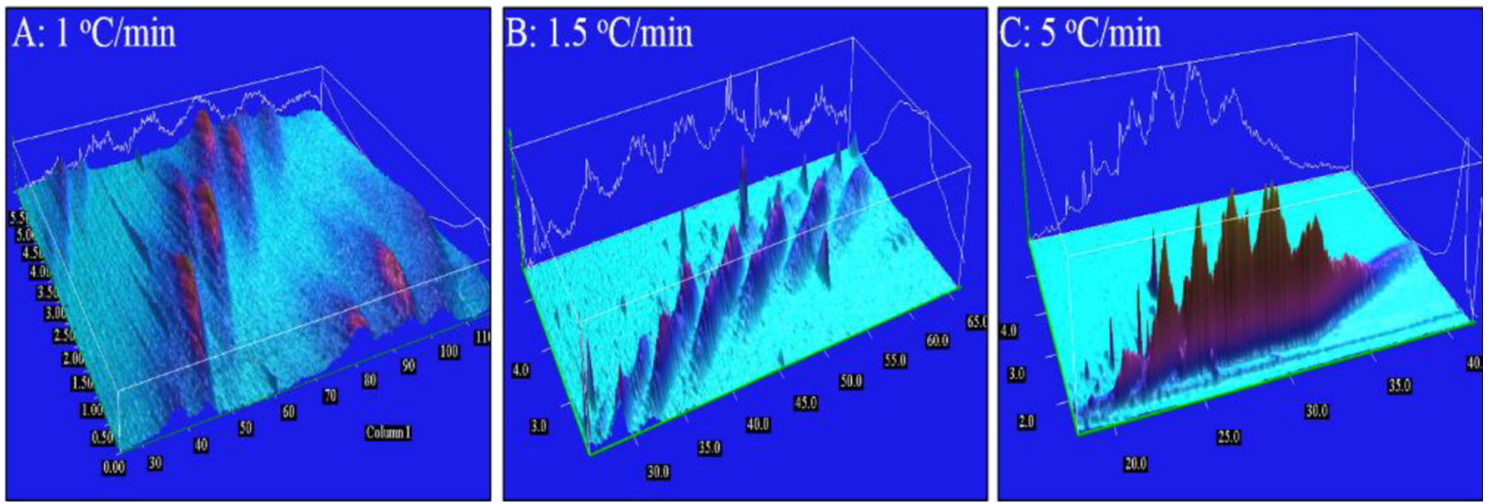

Fig. $2 \mathrm{GC} \times \mathrm{GC}-\mu \mathrm{ECD}$ chromatograms of a technical $\mathrm{C}_{10}-\mathrm{C}_{13} \mathrm{SCCP}$ mixture using different oven heating rates in the slow heating part of the oven temperature program: a $1{ }^{\circ} \mathrm{C} / \mathrm{min}, \mathbf{b} 1.5^{\circ} \mathrm{C} / \mathrm{min}$, and c $5^{\circ} \mathrm{C} / \mathrm{min}$

interfering compounds from the matrix can overlap with the target compound peaks and affect the ability to quantify the target compounds [26]. In other words, a modulation period must be chosen that preserves the first-dimension separation, which requires the peaks corresponding to all compounds eluted from the first GC column to be as sharp as possible. The SCCP congener separations were achieved using the DM$1 \times \mathrm{BPX}-50$ column combination and the chromatographic conditions described above, and modulation periods of 4,6 , and $8 \mathrm{~s}$ were evaluated; the results are shown in Fig. 3. Considerable wraparound of the SCCP congeners occurred when a modulation period of $4 \mathrm{~s}$ was used. We could safely conclude that a modulation period of $4 \mathrm{~s}$ was not adequate for the satisfactory separation of the SCCP congeners. Because of the large numbers of individual SCCP congeners and the selectivity of the BPX-50 column, the retention times of some of the compounds were longer than a modulation period of $4 \mathrm{~s}$, so they were not eluted within a single modulation cycle. This meant that some analytes may have been coeluted with compounds in a subsequent modulation cycle, destroying both the separation and the ordered structure of the 2D chromatogram. This situation could be improved by using a longer modulation period. A modulation period of $8 \mathrm{~s}$ gave poor separation performance, and the SCCPs were not effectively separated, as can be seen in chromatogram $\mathrm{C}$ in Fig. 3. The relatively long modulation period of $8 \mathrm{~s}$ allowed the peaks corresponding to the compounds that had already been separated in the first GC column to be recombined (or partly recombined) in the modulator before being injected into the second-dimension column. This decreased the number of peaks taken from the first-dimension column and effectively led to poorer chromatographic resolution being achieved using the first column. A modulation period of $6 \mathrm{~s}$ matched the highest analyte retention time on the second GC column and clearly gave good separation performance with no wraparound. A modulation period of $6 \mathrm{~s}$ gave an appropriate number of modulations per peak, so the separation achieved using the first column was preserved well after the second dimension of chromatography had been performed. Therefore, a modulation period of $6 \mathrm{~s}$ was chosen as the optimum. It should be noted that the optimum modulation period of $6 \mathrm{~s}$ reflects a compromise between the required sensitivity and the required resolution.

Optimization of the carrier gas flow rate and other $\mathrm{GC} \times \mathrm{GC}$ parameters

Optimizing a $\mathrm{GC} \times \mathrm{GC}$ system is not as simple as optimizing a conventional 1D-GC system. The two GC columns in a $\mathrm{GC} \times \mathrm{GC}$ system have different dimensions and are connected in series, so their carrier gas velocities cannot be selected independently, and a compromise is generally chosen that provides acceptable separation in both dimensions [24, 25].
Fig. $3 \mathrm{GC} \times \mathrm{GC}-\mu \mathrm{ECD}$ chromatograms of a technical $\mathrm{C}_{10}-\mathrm{C}_{13}$ SCCP mixture using three different modulation periods: $4 \mathrm{~s}(\mathbf{a}), 6 \mathrm{~s}(\mathbf{b})$, and $8 \mathrm{~s}(\mathbf{c})$
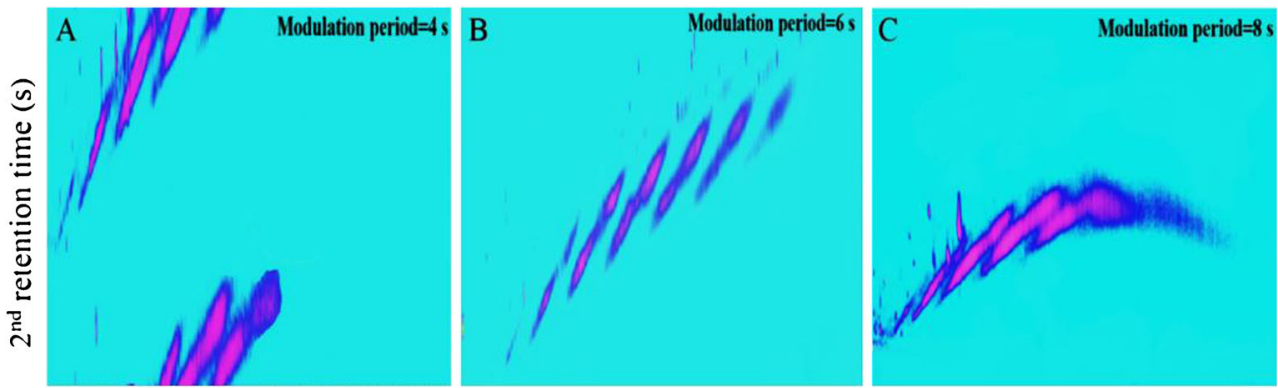

$1^{\text {st }}$ retention time $(\mathrm{min})$ 
Different carrier gas flow rates give different detector responses to the analyte peaks. A low carrier gas flow rate will result in a long analysis time, but a higher flow rate will require a higher head pressure, especially at higher temperatures, and this will have an adverse effect on the entire $\mathrm{GC} \times \mathrm{GC}$ system. We used a conventional flow rate of $1.0 \mathrm{~mL} / \mathrm{min}$. The very fast separation achieved using the second $\mathrm{GC}$ column means that $\mathrm{GC} \times \mathrm{GC}$ peaks will be extremely narrow, typically 50-600 ms at the baseline [27]. In this study, a 300-ms hot pulse was used so that sharp peaks were produced in the second dimension. A data acquisition rate of $50 \mathrm{~Hz}$ was used to avoid unnecessary band broadening. The modulator temperature also appeared to be important, and a modulator temperature of $280{ }^{\circ} \mathrm{C}$ appeared to be necessary to ensure that the hot jets efficiently released the trapped compounds during the modulation process and to avoid unnecessary band broadening.

\section{Analysis of SCCPs in technical mixtures}

Technical SCCP mixtures are composed of polychlorinated decanes, undecanes, dodecanes, and tridecanes. We validated the optimum parameters we had selected by analyzing four single carbon chain length $\left(\mathrm{C}_{10}, \mathrm{C}_{11}, \mathrm{C}_{12}\right.$, and $\left.\mathrm{C}_{13}\right) \mathrm{CP}$ mixtures, each with a chlorine content of $55 \%(\mathrm{w} / \mathrm{w})$. The $\mathrm{GC} \times \mathrm{GC}$ chromatograms of the four $\mathrm{CP}$ mixtures are shown in Fig. 4. The chromatogram of the polychlorinated decane mixture contained several parallel groups of peaks. The isomers lined up together as bands in the chromatogram, and the congener groups (or homologs) appeared as separate bands, as can be seen in Fig. 4a. The SCCPs were distributed in a "tiled" configuration, and the congeners were separated well on the basis of the number of chlorine substituents they contained. The isomers with different substitution patterns at each level of chlorination was marked using parallel lines. The colors in the chromatograms indicate that the pentachlorinated to heptachlorinated alkanes were the main components of the technical polychlorinated decane mixture. A good overall separation and an ordered structure, with parallel groups of peaks, were achieved using the system. We confirmed that tetrachlorinated to octachlorinated decanes were present and that the bands were for SCCPs that had been separated according to the number of chlorine substituents they contained by analyzing two standard mixtures of individual SCCP congeners. Similar separation patterns were found, i.e., on the basis of the number of chlorine substituents, for the other $\mathrm{CP}$ mixtures with single carbon chain lengths. The bands found for the polychlorinated undecanes were assigned to the trichlorinated to octachlorinated undecanes, as shown by the lines in Fig. 4b. The first-dimension retention times were longer for the polychlorinated undecanes than for the polychlorinated decanes, meaning that the polychlorinated undecanes were retained much more strongly than were the polychlorinated decanes. The polychlorinated dodecanes were eluted at longer retention times than were the polychlorinated undecanes, and the polychlorinated tridecanes were eluted even later. The increases in the retention times with the carbon chain length were caused by the boiling point increasing as the carbon chain length increased. We found that the $\mathrm{GC} \times \mathrm{GC}$ method that
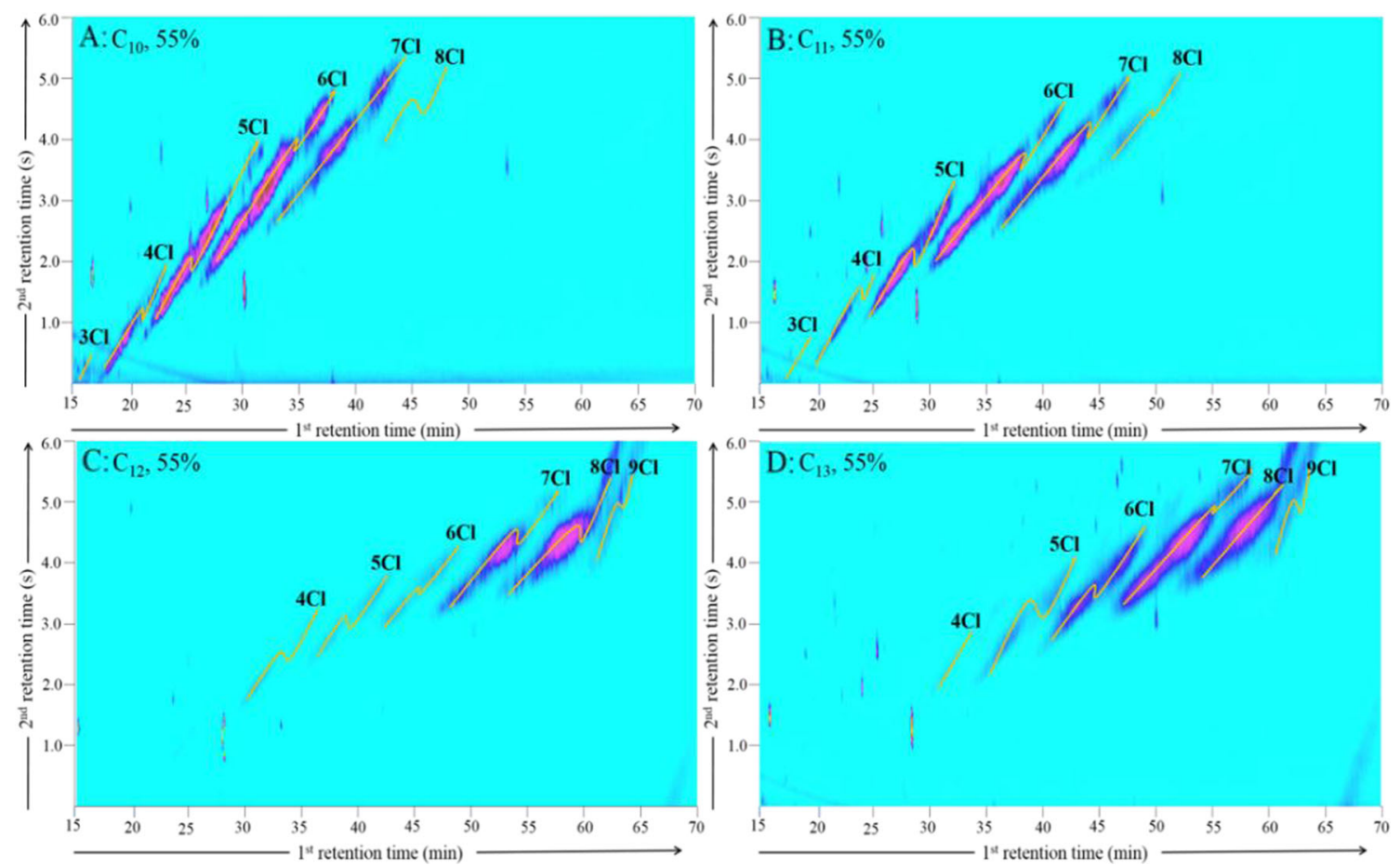

Fig. $4 \mathrm{GC} \times \mathrm{GC}-\mu \mathrm{ECD}$ chromatograms of a polychlorinated decanes, $\mathbf{b}$ polychlorinated undecanes, $\mathbf{c}$ polychlorinated dodecanes, and $\mathbf{d}$ polychlorinated tridecanes, all with a chlorine content of $55 \%(\mathrm{w} / \mathrm{w})$ 
had been developed provided baseline separation of the SCCP components with the same carbon chain length into groups according to the number of chlorine substituents they contained.

The SCCP congeners with different carbon chain lengths and different numbers of chlorine substituents in a $\mathrm{C}_{10}-\mathrm{C}_{13}$ technical mixture that was analyzed using the method we had developed are shown in the chromatogram in Fig. 5b. The SCCP congeners were separated in the chromatogram rather well, and the overall separation achieved was much better than has been achieved using 1D GC. The orthogonality of the column combination meant that the separation in the first dimension was mainly determined by the volatilities of the components, and the separation in the second dimension was mainly determined by the polarities of the components. As the length of the carbon skeleton increased, the boiling points of the components increased and the retention time in the first dimension increased. An interesting observation is that ordered structures were found in the chromatogram that comprised compounds with the same number of carbon atoms plus chlorine atoms, as shown in Fig. 5b. This means that one carbon atom contributed about the same increase in retention time as did one chlorine atom [20]. In other words, compounds with the same number of carbon atoms plus chlorine atoms were found at the same position on the diagonal lines in the $2 \mathrm{D}$ chromatogram. The positions of different homologs are indicated with lines of different colors in Fig. 5b. For example, $\mathrm{C}_{10} \mathrm{Cl}_{7}, \mathrm{C}_{11} \mathrm{Cl}_{6}, \mathrm{C}_{12} \mathrm{Cl}_{5}$, and $\mathrm{C}_{13} \mathrm{Cl}_{4}$ all appeared on the same diagonal line, and $\mathrm{C}_{12} \mathrm{Cl}_{8}$ and $\mathrm{C}_{13} \mathrm{Cl}_{7}$ fell on the same line. Combined with the previous finding relating to $\mathrm{CPs}$ with single carbon chain lengths, it appears that congeners with the same number of carbon atoms but different numbers of chlorine atoms will appear as parallel diagonal bands in the $\mathrm{GC} \times \mathrm{GC}$ chromatogram. Therefore, we can safely conclude that the position of the various compounds in each diagonal band depends on the number of carbon atoms, compounds with longer carbon chains having lower second-dimension retention times. This selectivity based on carbon chain length helps to create a distinct separation pattern for compounds that differ by at least three carbon atoms. The great improvement in the separation of SCCPs when using $\mathrm{GC} \times \mathrm{GC}$ rather than $1 \mathrm{D}$ GC (Fig. 5a) means that the method we have developed offers a new way of analyzing SCCPs in environmental samples.

Comparison of the SCCP separation achieved using 1D GC and $\mathrm{GC} \times \mathrm{GC}$

The separation performance and the LODs achieved using 1D $\mathrm{GC}$ and $\mathrm{GC} \times \mathrm{GC}$ using the same type of $\mathrm{GC}$ system and identical conditions (such as the carrier gas flow rate and oven temperature program) were compared to specifically demonstrate the difference between the separation performances of $1 \mathrm{D} \mathrm{GC}$ and GC $\times$ GC. As shown in Fig. 5a, the 1D chromatogram of a technical SCCP mixture contained a characteristic unresolved, broad feature that clearly indicated that there were a very large number of coeluted compounds and partly overlapping peaks. In contrast, the $\mathrm{GC} \times \mathrm{GC}$ system was able to separate groups of SCCP congeners (Fig. 5b). In terms of separation of the components, therefore, $\mathrm{GC} \times \mathrm{GC}$ is a better
Fig. 5 Chromatograms of a technical $\mathrm{C}_{10}-\mathrm{C}_{13}$ SCCP mixture with a $55 \%(\mathrm{w} / \mathrm{w})$ chlorine content obtained using a gas chromatography-electron capture detection $(E C D)$ and $\mathbf{b} \mathrm{GC} \times \mathrm{GC}-$ $\mu \mathrm{ECD}$
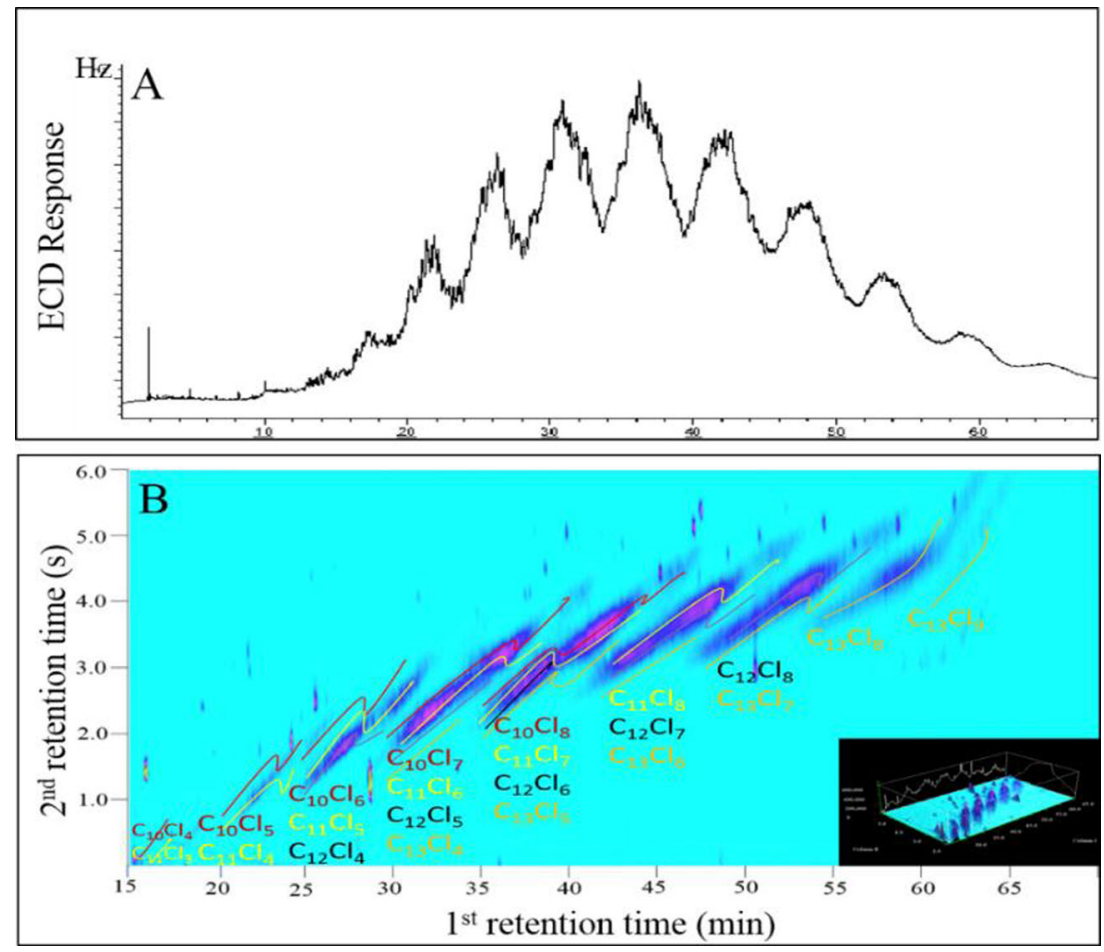
Table 1 Limits of detection $(L O D)$ for selected short-chain chlorinated paraffin congeners obtained using a gas chromatography $(G C)$-electron capture detection $(E C D)$ system and the two-dimensional GC $(G C \times G C)$-micro-ECD $(\mu E C D)$ system

\begin{tabular}{llll}
\hline Name & Formula & LOD $(\mathrm{pg} / \mathrm{L})$ & $\mathrm{GC} \times \mathrm{GC}-\mu \mathrm{ECD}$ \\
\cline { 2 - 4 } & & $\mathrm{GC}-\mathrm{ECD}$ & 5 \\
$1,2,5,6,9$-Pentachlorodecane & $\mathrm{C}_{10} \mathrm{Cl}_{5}$ & 15 & 5 \\
$1,2,4,5,9,10$-Hexachlorodecane & $\mathrm{C}_{10} \mathrm{Cl}_{6}$ & 15 & 3 \\
$1,2,4,5,6,9,10$-Heptachlorodecane & $\mathrm{C}_{10} \mathrm{Cl}_{7}$ & 10 & 1 \\
2,3,4,5,6,7,8,9-Octachlorodecane & $\mathrm{C}_{10} \mathrm{Cl}_{8}$ & 4 & 1 \\
\hline
\end{tabular}

The LOD was defined as the concentration giving a peak with a signal-to-noise ratio of 3:1.

choice than 1D GC for separating and screening SCCP congeners in environmental samples. The LODs that were achieved for selected SCCP congeners using a GC-ECD system and the $\mathrm{GC} \times \mathrm{GC}-\mu \mathrm{ECD}$ system were estimated. It is not straightforward to determine LODs for a $\mathrm{GC} \times \mathrm{GC}$ system, and this has been discussed elsewhere $[15,28]$. The LODs (at a signal-to-noise ratio of 3:1) were calculated as follows: a series of increasingly dilute standards containing mixtures of selected SCCP congeners were injected and the LOD was determined using the chromatogram that contained only one peak (or, occasionally, two equally intense peaks) and in which the other, smaller, peaks had disappeared. The LODs that were found are shown in Table 1. It can be seen that, with use of the optimized conditions described above, the LODs were three to four times lower for the $\mathrm{GC} \times \mathrm{GC}$ system than for the $1 \mathrm{D}-\mathrm{GC}$ system. An LOD of $5 \mathrm{pg} / \mathrm{L}$ or better can be expected for pentachlorinated or more highly chlorinated SCCPs using comprehensive $\mathrm{GC} \times \mathrm{GC}$ with a $\mu \mathrm{ECD}$.

Analysis of SCCPs in fish extracts

We demonstrated the feasibility of the method we had developed for separating and quickly screening SCCPs in real complex environmental samples by analyzing extracts of a fish sample. The fish sample had been collected from a river in China, and it was prepared for analysis as described in "Sample preparation." The extract was then analyzed using the $\mathrm{GC} \times \mathrm{GC}-\mu \mathrm{ECD}$ method we had established. The resulting chromatogram of the fish sample is shown in Fig. 6, and it can be seen that a large band of SCCP congeners and numerous additional compounds were detected. The presence of a large number of SCCPs and other organochlorine compounds is clear from the chromatogram, and more than 1,500 peaks could be distinguished. It was clear that the SCCPs had been separated into groups, and they were found to have been separated depending on the number of carbon atoms and the number of chlorine atoms they contained. Further, the SCCPs had been separated well from coextracted potentially interfering compounds. According to the responses determined using the $\mathrm{GC} \times \mathrm{GC}$ software, the fish extract contained high SCCP concentrations, which suggests that the fish sample was probably contaminated with SCCPs. The Triplophysa siluroides that was analyzed is a resident and relatively long-lived fish species, so we can safely conclude that SCCPs bioaccumulate in biota in these area.
Fig. $6 \mathrm{GC} \times \mathrm{GC}-\mu \mathrm{ECD}$

chromatogram of a fish tissue extract. The fish sample (Triplophysa siluroides) was collected from a river in China. $\mathrm{CBz}$ chlorobenzenes, $O C P S$ organochlorine pesticides

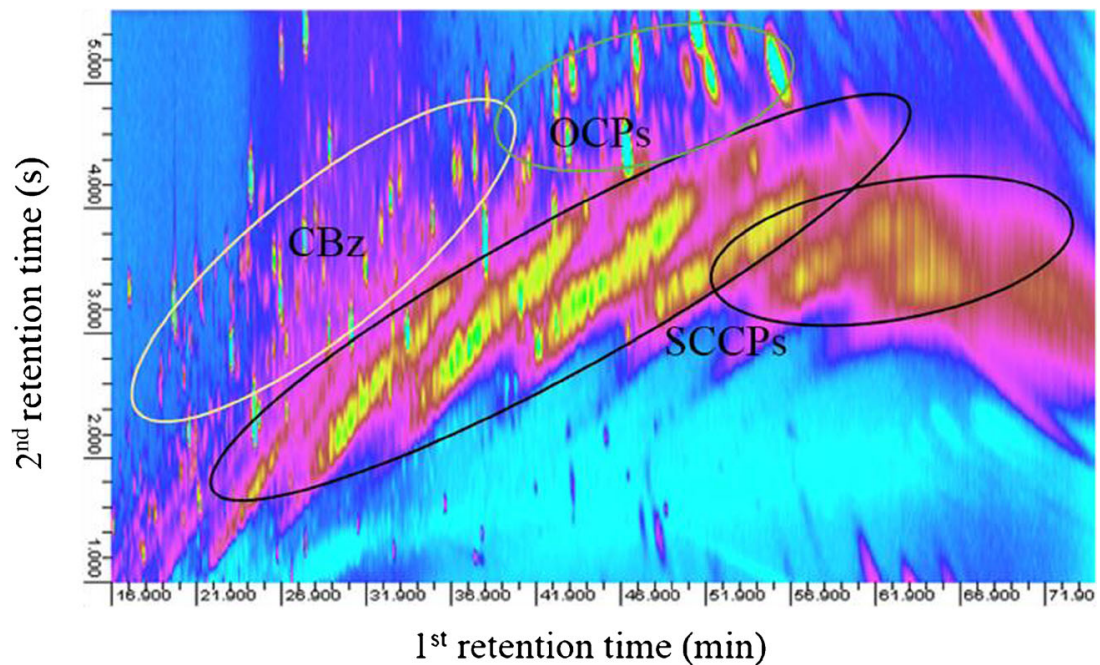


The method we developed not only separated SCCPs into groups of congeners depending on the carbon chain length and the number of chlorine substituents in the component, but also gave good separation of the SCCP groups from other organochlorine compounds. In addition to separating and quickly screening SCCPs in a fish sample, this 2D technique can also be used to detect other classes of contaminants in the sample. Chlorobenzenes and organochlorine pesticides were found to be ubiquitous in the fish samples, as shown in Fig. 6. These organochlorine compounds often interfere with the detection of SCCPs when using 1D GC. The separation of the SCCPs from other compounds means that the $\mathrm{GC} \times \mathrm{GC}$ method can be used to determine the effectiveness of a sample pretreatment method, and means that the determination of the SCCPs and the other compounds in real environmental samples will be more accurate than it would be otherwise. Overall, it can be concluded that the $\mathrm{GC} \times \mathrm{GC}$ system allows much better separation of a wide range of halogenated organic compounds, including SCCPs, to be achieved than could be achieved using 1D GC.

\section{Conclusions}

The separation and screening of SCCPs by comprehensive $\mathrm{GC} \times \mathrm{GC}$ was studied. The method developed was used for the analysis of SCCPs in technical mixtures and biological samples. The GC parameters influencing the separation process, such as column set, GC oven temperature program, modulation period, and other instrumental parameters, were optimized. The DM-1×BPX-50 column combination separated the SCCP congeners well. SCCP congeners with the same carbon chain length were separated depending on the number of chlorine substituents they contained. For a technical SCCP mixture containing SCCP congeners with different carbon chain lengths and different numbers of chlorine substituents, the congeners were separated into those with the same number of carbon atoms plus chlorine atoms. The $\mathrm{GC} \times \mathrm{GC}$ system exhibited better separation than traditional 1D GC of not only the SCCP congeners but also the SCCPs and other compounds. The results obtained from analyzing a fish sample showed that the $\mathrm{GC} \times \mathrm{GC}-\mu \mathrm{ECD}$ system has great potential for screening SCCPs in real environmental samples, with good separation and excellent sensitivity. More than 1,500 compounds, including SCCP congeners and other organochlorine compounds, were identified in the fish extract using a 75-min $\mathrm{GC} \times \mathrm{GC}$ run. The LODs for selected SCCP congeners obtained using the $\mathrm{GC} \times \mathrm{GC}-\mu \mathrm{ECD}$ system were between 1 and $5 \mathrm{pg} / \mathrm{L}$, much lower than the GC-ECD system offered. This study shows that $\mathrm{GC} \times \mathrm{GC}$ has been proven to be a good technique for separating very complex mixtures, and it may also be a new method to analyze other complex environmental pollutants. Research on SCCPs using $\mathrm{GC} \times \mathrm{GC}$ will continue, and the analyzing of SCCPs in different types of samples by $\mathrm{GC} \times \mathrm{GC}$ will be studied further.

Acknowledgment This research was funded by the National Natural Science Foundation of China (nos. 21377140, 21321004, and 21361140359).

\section{References}

1. Tomy GT, Stern GA, Lockhart WL, Muir DCG (1999) Environ Sci Technol 33:2858-2863

2. Zeng LX, Wang T, Han WY, Yuan B, Liu QA, Wang YW, Jiang GB (2011) Environ Sci Technol 45:2100-2106

3. Eljarrat E, Barcelo D (2006) Trends Anal Chem 25:421-434

4. Marvin CH, Painter S, Tomy GT, Stern GA, Braekevelt E, Muir DCG (2003) Environ Sci Technol 37:4561-4568

5. Stockholm Convention on Persistent Organic Pollutants (2009). http://chm.pops.int. Accessed 30 Jun 2014

6. Castells P, Parera J, Santos FJ, Galceran MT (2008) Chemosphere 70: $1552-1562$

7. Ma X, Chen C, Zhang H, Gao Y, Wang Z, Yao Z, Chen J, Chen J (2014) Mar Pollut Bull 79:299-304

8. Wang T, Wang Y, Jiang G (2013) Environ Sci Technol 47:11924 11925

9. Tomy GT, Stern GA, Muir DCG, Fisk AT, Cymbalisty CD, Westmore JB (1997) Anal Chem 69:2762-2771

10. Peters AJ, Tomy GT, Jones KC, Coleman P, Stern GA (1999) Atmos Environ 34:3085-3090

11. Reth M, Oehme M (2004) Anal Bioanal Chem 378:1741-1747

12. Xu X, van Stee LLP, Williams J, Beens J, Adahchour M, Vreuls RJJ, Brinkman UAT, Lelieveld J (2003) Atmos Chem Phys 3:665-682

13. Harju M, Danielsson C, Haglund P (2003) J Chromatogr A 1019:111126

14. Kristenson EM, Korytár P, Danielsson C, Kallio M, Brandt M, Mäkelä J, Vreuls RJJ, Beens J, Brinkman UAT (2003) J Chromatogr A 1019:65-77

15. Korytár P, Leonards PEG, de Boer J, Brinkman UAT (2002) J Chromatogr A 958:203-218

16. Korytár P, Danielsson C, Leonards PEG, Haglund P, de Boer J, Brinkman UAT (2004) J Chromatogr A 1038:189-199

17. Haglund P, Korytár P, Danielsson C, Diaz J, Wiberg K, Leonards P, Brinkman UAT, de Boer J (2008) Anal Bioanal Chem 390:1815-1827

18. Muscalu AM, Reiner EJ, Liss SN, Chen T, Ladwig G, Morse D (2011) Anal Bioanal Chem 401:2403-2413

19. Zhang B, Zheng MH, Liu GR, Li C, Gao LR (2012) Chin J Anal Chem 40:1213-1218

20. Korytár P, Parera J, Leonards PEG, Santos FJ, de Boer J, Brinkman UAT (2005) J Chromatogr A 1086:71-82

21. Gao Y, Zhang H, Chen J, Zhang Q, Tian Y, Qi P, Yu Z (2011) Anal Chim Acta 703:187-193

22. Korytár P, Haglund P, de Boer J, Brinkman UAT (2006) Trends Anal Chem 25:373-396

23. Omar J, Alonso I, Olivares M, Vallejo A, Etxebarria N (2012) Talanta $88: 145-151$

24. Mostafa A, Edwards M, Gorecki T (2012) J Chromatogr A 1255:38-55

25. Dallüge J, Beens J, Brinkman UAT (2003) J Chromatogr A 1000:69108

26. Adahchour M, Beens J, Vreuls RJJ, Brinkman UAT (2006) Trends Anal Chem 25:540-553

27. Adahchour M, Beens J, Brinkman UAT (2008) J Chromatogr A 1186:67-108 
28. Dalluge J, Vreuls RJJ, Beens J, Brinkman UAT (2002) J Sep Sci 25: 201-214

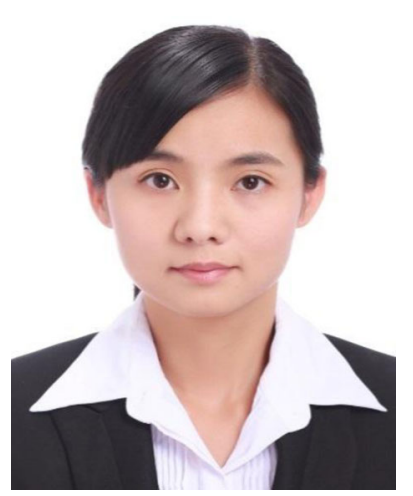

Dan Xia is a graduate student at the State Key Laboratory of Environmental Chemistry and Ecotoxicology, Research Center for EcoEnvironmental Sciences, Chinese Academy of Sciences. She majored in environmental analytical chemistry, and her research is focused on new methods for analyzing trace amounts of persistent organic pollutants using comprehensive two-dimensional gas chromatography.

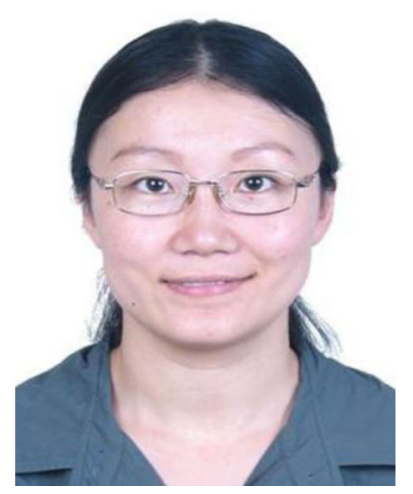

Lirong Gao is an associate professor at the State Key Laboratory of Environmental Chemistry and Ecotoxicology, Research Center for Eco-Environmental Sciences, Chinese Academy of Sciences. She majored in analytical methods and the environmental fate and transport of persistent organic pollutants (POPs), and her research is mainly focused on new methods for analyzing emerging POPs, and quality assurance and quality control in analyses of POPs.

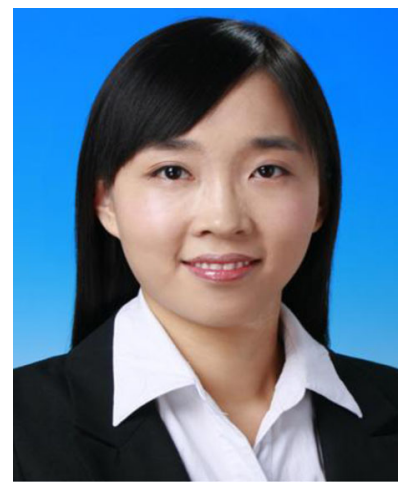

Shuai Zhu is a graduate student at the State Key Laboratory of Environmental Chemistry and Ecotoxicology, Research Center for Eco-Environmental Sciences, Chinese Academy of Sciences. She majored in environmental analytical chemistry, and her research is mainly focused on determining toxaphene in environmental samples by using comprehensive two-dimensional gas chromatography.

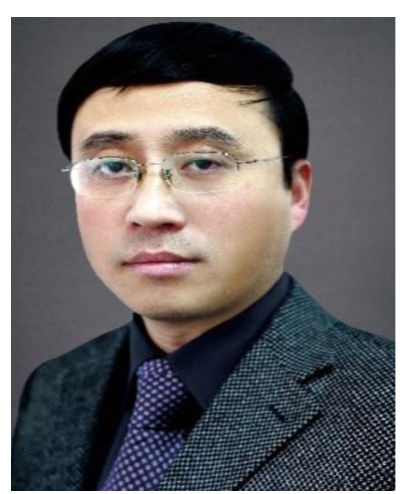

Minghui Zheng is Deputy Director of the State Key Laboratory of Environmental Chemistry and Ecotoxicology, Research Center for Eco-Environmental Sciences, Chinese Academy of Sciences. He is a leading expert on monitoring of persistent organic pollutants (POPs) and the study of dioxins in China. He also contributes to global POP monitoring activities. $\mathrm{He}$ is a coordinator of the Asia-Pacific regional organization group on POP monitoring and is a member of the Global Coordination Group on POP monitoring under the Stockholm Convention. He has published more than 150 scientific articles. 